\title{
Mid-Term Outcomes and Angiographic Patency of Redo Coronary Artery Bypass Grafting: A Comparison between Off- Pump and On-Pump Surgery
}

\author{
Suk Ho Sohn, M.D., Seung Hyun Kim, M.D., Ho Young Hwang, M.D., Ph.D., Ki-Bong Kim, M.D., Ph.D. \\ Department of Thoracic and Cardiovascular Surgery, Seoul National University Hospital, Seoul National University College of Medicine, Seoul, Korea
}

\author{
ARTICLE INFO \\ Received September 23, 2020 \\ Revised November 11, 2020 \\ Accepted November 17, 2020 \\ Corresponding author \\ Ki-Bong Kim \\ Tel $82-2-2072-3482$ \\ Fax 82-2-747-5245 \\ E-mail kimkb@snu.ac.kr \\ ORCID \\ https://orcid.org/0000-0002-4918-7262
}

\begin{abstract}
Background: We evaluated the mid-term outcomes and angiographic patency of redo coronary artery bypass grafting (CABG).

Methods: Of 2,851 patients who underwent isolated CABG at Seoul National University Hospital from 2000 to 2017, 88 underwent redo CABG. Patients' mean age at redo CABG was $66.0 \pm 8.0$ years. The mean interval between the first-time and redo CABG was 113.0 \pm 62.4 months. The mean follow-up duration was 86 months. Early and mid-term clinical outcomes were evaluated. Angiographic patency rates were evaluated early (1-2 days), 1 year, and 5 years after surgery. Comparative analyses between on-pump and off-pump CABG were also performed.

Results: The culprits for reoperation were previous grafts (65.6\%), native coronary vessels (17.8\%), and both (16.7\%). Off-pump CABG was performed in 75 cases (85.2\%), and the mean number of distal anastomoses was $1.8 \pm 0.8$. The saphenous vein (39.7\%) was used most frequently, followed by the right internal thoracic artery (28.4\%), right gastroepiploic artery (21.3\%), left internal thoracic artery (7.8\%), and radial artery (2.8\%). Operative mortality was $1.1 \%$. The overall survival, cumulative incidence of cardiac death, and cumulative incidence of major adverse cardiac events were $71.3 \%, 12.0 \%$, and $23.3 \%$ at 5 years after surgery, respectively. The overall angiographic patency rates were $95.7 \%, 90.1 \%$, and $92.2 \%$ on early, 1 -year, and 5-year angiograms, respectively. The angiographic patency rates of saphenous vein grafts were $93.1 \%, 85.6 \%$, and $91.3 \%$ on early, 1-year, and 5-year angiograms, respectively. No significant differences in clinical outcomes or angiographic patency rates were observed between the on-pump ( $n=13)$ versus off-pump $(n=75)$ groups. Multivariable analysis revealed that age (hazard ratio [HR], 1.07; $p=0.005$ ) and chronic kidney disease $(H R, 3.85 ; p=0.001)$ were risk factors for all-cause mortality.

Conclusion: Redo CABG could mostly be performed using the off-pump technique and did not show increased operative mortality and morbidities.
\end{abstract}

Keywords: Coronary artery bypass, Reoperation, Coronary angiography

\section{Introduction}

It has been estimated that $10 \%-20 \%$ of patients with previous coronary artery bypass grafting $(\mathrm{CABG})$ require a second revascularization procedure within 10 years due to the progression of atherosclerosis $[1,2]$. For these patients, both the American (2011) and European (2014) guidelines suggest percutaneous coronary intervention (PCI) as the first-line treatment for repeat revascularization $[3,4]$, while the European (2018) guidelines recommend redo CABG only for patients without a patent internal mammary artery (IMA) graft to the left anterior descending artery (LAD) [5].

Currently, we are confronted with a pronounced drop in the number of redo CABGs, as well as a decrease in the number of isolated CABGs. One reason for this decrease is that redo $C A B G$ is one of the most challenging cardiac surgical procedures. The major factors that make these operations very complicated are sternal reentry and intrapericardial dissection, which should be performed to avoid in- 
jury to the patent grafts; poor access to the target vessels, which usually lie on the thickened epicardium; a shortage of available grafts owing to a previous operation; and suboptimal cardiac function with other comorbidities [6,7]. In addition, some surgeons are unwilling to perform redo CABG because it is known to be associated with increased early mortality, with reported rates as high as $16 \%$ [8]. Finally, optimal medical therapy with antiplatelets and statins [9], advancements in PCI, and the progress in surgical techniques with the use of the IMA during first-time CABG have contributed to the reduced frequency of redo CABG [6].

Previous studies have reported higher surgical complexity and inferior clinical outcomes, with increased operative mortality and morbidities, in redo CABG than in firsttime CABG; however, some other studies have shown improvements in hospital mortality rates after redo CABG, despite deterioration in patients' risk profiles $[6,10,11]$. Furthermore, few studies have described the long-term outcomes of redo CABG patients in the recent era.

Thus, the aims of the present study were (1) to evaluate the early- and mid-term clinical outcomes and angiographic patency of redo CABG and (2) to compare the outcomes between on-pump and off-pump redo CABG.

\section{Methods}

The study protocol was reviewed by the Institutional Review Board of Seoul National University Hospital and was approved as a minimal-risk retrospective study (approval no., H-2006-195-1138) that did not require individual consent based on the institutional guidelines for waiving consent.

\section{Patient characteristics}

Of 2,851 patients who underwent isolated CABG between 2000 and 2017 in Seoul National University Hospital, redo $C A B G$, which was defined as a repeat CABG performed at least 30 days after the previous CABG, was performed in 90 patients (3.2\%) (Fig. 1). Among 90 redo CABG patients, 2 patients who underwent a second redo CABG were excluded from the analysis, and a total of 88 patients were included in this study. The mean age of the patients at the time of redo CABG was $66.0 \pm 8.0$ years. Hypertension (66.7\%), diabetes mellitus (57.8\%), and dyslipidemia (33.3\%) were the most frequently observed comorbidities in the overall population. Previous PCI was performed in 34 patients (37.8\%); among them, 20 patients received PCI in the period between the first-time and the redo CABGs. The culprit lesions leading to redo CABG were graft occlusion (65.6\%), native disease progression (17.8\%), and both (16.7\%). The time interval between the first-time and redo operations was 113.0 \pm 62.4 months (Table 1).

\section{Surgical techniques and revascularization strategies}

The basic surgical techniques for redo CABG followed the institutional principles of CABG as previously described [12]. Most of the operations were performed by a single surgeon (K.B.K.). Off-pump CABG was initially attempted in most of the patients; in some patients, however, cardiopulmonary bypass was required during the operation due to hemodynamic instability or poor exposure caused by severe adhesions. Intraoperative intra-aortic balloon pump (IABP) therapy was performed in patients who

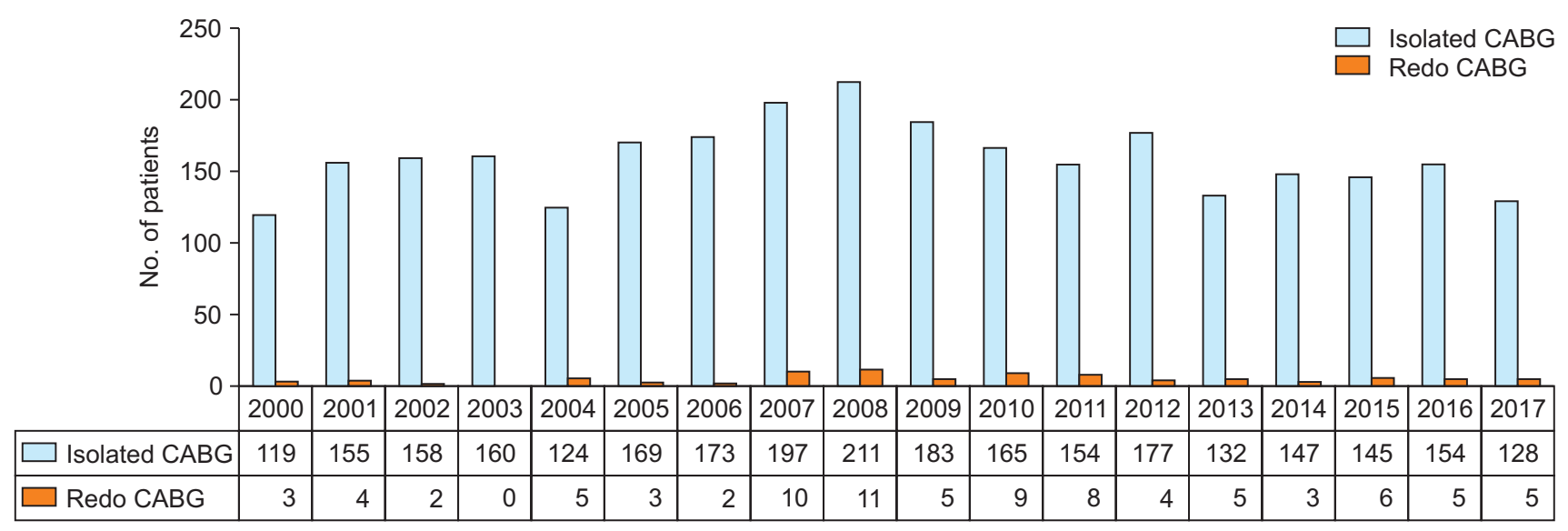

Fig. 1. Number of overall isolated CABG and redo CABG procedures performed from 2000 to 2017 at Seoul National University Hospital. CABG, coronary artery bypass grafting. 
Table 1. Preoperative characteristics and risk factors of the study population

\begin{tabular}{|c|c|c|c|c|}
\hline Characteristic & Total $(n=88)$ & On-pump (n=13) & Off-pump ( $\mathrm{n}=75)$ & $p$-value \\
\hline Age (yr) & $66.0 \pm 8.0$ & $67.0 \pm 6.1$ & $65.9 \pm 8.3$ & 0.629 \\
\hline Female & $22(25.0)$ & $2(15.4)$ & $20(26.7)$ & 0.504 \\
\hline Body surface area $\left(\mathrm{m}^{2}\right)$ & $1.71 \pm 0.16$ & $1.66 \pm 0.22$ & $1.72 \pm 0.15$ & 0.253 \\
\hline Body mass index $\left(\mathrm{kg} / \mathrm{m}^{2}\right)$ & $24.6 \pm 2.9$ & $23.8 \pm 3.4$ & $24.8 \pm 2.8$ & 0.259 \\
\hline \multicolumn{5}{|l|}{ Risk factors } \\
\hline Smoking & $27(30.7)$ & $5(38.5)$ & $22(29.3)$ & 0.527 \\
\hline Hypertension & $58(65.9)$ & $10(76.9)$ & $48(64.0)$ & 0.529 \\
\hline Diabetes mellitus & $51(58.0)$ & $9(69.2)$ & $42(56.0)$ & 0.372 \\
\hline Dyslipidemia & $30(34.1)$ & $6(46.2)$ & $24(32.0)$ & 0.353 \\
\hline History of stroke & $14(15.9)$ & $4(30.8)$ & $10(13.3)$ & 0.210 \\
\hline Chronic kidney disease & $14(15.9)$ & 0 & $14(18.7)$ & 0.117 \\
\hline LVEF $<35 \%$ & $10(11.4)$ & $1(7.7)$ & $9(12.0)$ & $>0.999$ \\
\hline \multicolumn{5}{|l|}{ Preoperative diagnosis } \\
\hline Unstable angina & $54(61.4)$ & $6(46.2)$ & $48(64.0)$ & 0.222 \\
\hline Stable angina & $24(27.3)$ & $5(38.5)$ & $19(25.3)$ & 0.330 \\
\hline Postinfarct angina & $10(11.4)$ & $2(15.4)$ & $8(10.7)$ & 0.638 \\
\hline \multicolumn{5}{|l|}{ Type of vessel disease } \\
\hline LMD+3VD & $7(8.0)$ & $1(7.7)$ & $6(8.0)$ & $>0.999$ \\
\hline LMD+2VD & $12(13.6)$ & $3(23.1)$ & $9(12.0)$ & 0.375 \\
\hline LMD+1VD & $2(2.3)$ & 0 & $2(2.7)$ & $>0.999$ \\
\hline LMD & $1(1.1)$ & 0 & $1(1.3)$ & $>0.999$ \\
\hline $3 V D$ & $14(15.9)$ & 0 & $14(18.7)$ & 0.117 \\
\hline 2VD & $33(37.5)$ & $7(53.8)$ & $26(34.7)$ & 0.223 \\
\hline 1VD & $19(21.6)$ & $2(15.4)$ & $17(22.7)$ & 0.726 \\
\hline History of PCI & $33(37.5)$ & $5(38.5)$ & $28(37.3)$ & $>0.999$ \\
\hline $\mathrm{PCl}$ after first $\mathrm{CABG}$ & $20(22.2)$ & $3(20.0)$ & $17(22.7)$ & 0.562 \\
\hline Culprit for reoperation & & & & 0.319 \\
\hline Graft & $58(65.9)$ & $7(53.8)$ & $51(68.0)$ & \\
\hline Native vessel & $16(18.2)$ & $2(15.4)$ & $14(18.7)$ & \\
\hline Both (graft+native vessel) & $14(15.9)$ & $4(30.8)$ & $10(13.3)$ & \\
\hline Interval between operations (mo) & $113.0 \pm 62.4$ & $87.5 \pm 69.2$ & $117.5 \pm 60.6$ & 0.110 \\
\hline
\end{tabular}

Values are presented as mean \pm standard deviation or number (\%).

LVEF, left ventricular ejection fraction; LMD, left main disease; VD, vessel disease; PCl, percutaneous intervention; CABG, coronary artery bypass grafting.

developed hemodynamic instability during cardiac manipulation or coronary anastomosis although adhesiolysis had been completed without cardiopulmonary bypass. The target coronary arteries for bypass grafting were determined by a comprehensive evaluation of the patient, including serial angiographic results, myocardial single-photon emission computed tomography, echocardiography, and symptoms. The choice of the graft to use for redo CABG was determined based on (1) excluding previously used grafts, (2) the number and location of the target coronary arteries, and (3) the surgeon's preference. All anastomoses were performed using a high-power magnification loupe $(\times 4.5$ magnification) and checked with a flowmeter after completion of revascularization and just before pericardial closure.
All the patients received aspirin therapy (100 mg daily) until the day of surgery and resumed the therapy as soon as possible after surgery, usually 1 day postoperatively. Clopidogrel (75 mg daily) was combined simultaneously with aspirin for 1 year postoperatively. For patients with high serum levels of low-density lipoprotein cholesterol $(>100 \mathrm{mg} / \mathrm{dL})$, drug therapy was started.

\section{Evaluation of clinical outcomes}

Operative mortality was defined as any death within 30 days after surgery or during the same hospital admission. After discharge, all patients regularly received postoperative follow-up at the outpatient clinic at 3- or 4-month intervals and were interviewed by telephone to confirm their 
condition if the last clinic visit had not been conducted as scheduled. For patients who were lost to follow-up, national health insurance data and information from Statistics Korea, a central organization for statistics under the Ministry of Strategy and Finance, were utilized to confirm their survival or their date of death. The clinical and angiographic follow-up was closed on June 30, 2020. The follow-up data were complete in $98.9 \%$ (87 of 88 ) of patients, with a mean follow-up duration of $85.7 \pm 55.8$ months.

Cardiac death was defined as any death related to cardiac events, including sudden death during follow-up. Major adverse cardiac events (MACE) included acute myocardial infarction, coronary reintervention, and cardiac death.

\section{Evaluation of angiographic patency}

Early, 1-year, and 5-year follow-up coronary angiograms were performed regardless of the patients' anginal symptoms. Patients who died, refused angiographic evaluation, or had renal function impairment were excluded from angiographic follow-up. However, patients receiving renal replacement therapy were included in the angiographic follow-up.

Early (1 day; interquartile range [IQR], 1-2 months) patency rates were evaluated by coronary angiograms in $100.0 \%$ (88 of 88 ) of the patients. One-year (12.6 months; IQR, 12.0-13.5 months) and 5-year (62.3 months; IQR, 60.4-70.1 months) angiograms were performed in $87.5 \%$ and $54.5 \%$ of the patients, respectively. Patients who were reluctant to undergo coronary angiography received multidetector computed tomography (MDCT) angiography as an alternative evaluation method. Twenty-five and 20 patients underwent MDCT angiography instead of coronary angiography for their 1-year and 5-year patency evaluations, respectively. If an MDCT examination suggested occluded or stenotic grafts when compared with the patient's previous angiograms, coronary angiography was then performed to confirm the status of graft patency. Two blinded specialists reviewed all coronary angiograms, and 2 specialists reviewed all MDCT angiograms. Each set of specialists reached a consensus in their interpretations of graft patency. Graft patency was graded in the manner described by FitzGibbon et al. [13]. Grade A (excellent) and B (fair) grafts were considered to be patent. Grade O grafts, which included stenosis of $\geq 75 \%$ of the grafted coronary artery or a totally occluded graft, were classified as occluded.

Operative data included graft usage, grafting strategy, the graft for target vessel anastomosis, the target native coronary artery (LAD, diagonal branch, obtuse marginal artery $[\mathrm{OM}]$, right coronary artery $[\mathrm{RCA}]$, posterior descending artery [PDA], and posterolateral branch [PLB]), and revascularized areas of the heart (LAD, left circumflex artery [LCx], or RCA territory), and whether complete revascularization had been accomplished. Complete revascularization was defined as accomplishment of distal anastomoses to each territory with angiographically significant stenosis.

\section{Statistical analysis}

Statistical analysis was conducted with IBM SPSS software ver. 25.0 (IBM Corp., Armonk, NY, USA) and SAS software ver. 9.3 (SAS Institute Inc., Cary, NC, USA). Continuous variables are presented as the mean \pm standard deviation for normally distributed data or as the median with interquartile range for data that were not normally distributed, while categorical variables are presented as the number and percentage of subjects. Comparisons of baseline demographics, operative data, and early clinical outcomes between the 2 groups were performed using the chi-square test or Fisher exact test for categorical variables, the Student t-test or Wilcoxon rank sum test for continuous variables, and the generalized estimating equation (GEE) method for operative data with multiple data points per subject. Overall survival was estimated using the Kaplan-Meier method, and comparisons between the 2 groups were performed using a Cox proportional-hazard model. For the analysis of cardiac death and MACE, cumulative incidence curves were estimated while considering competing events. Noncardiac death was considered as a competing event for cardiac death and MACE. A Fine and Gray regression model was used to compare cumulative incidence between the groups [14]. The proportional hazards assumption was checked by log-minus-log plots of survival functions for categorical variables or time-dependent covariates in the Cox model and the Fine and Gray model. Restricted cubic splines were used to check the assumption of linearity between each continuous variable and its log hazard. For risk factor analysis, preoperative variables and operative factors with a $p$-value of $<0.1$ in the univariate analysis were included in the multivariable analysis, and these variables were also used to adjust the comparison of mid-term outcomes. The graft patency rates were compared using logistic regression with GEE to account for multiple measurements per subject or using a logistic regression with the Firth penalized-likelihood bias-reduction method in cases where GEE failed to converge because 1 of the groups (the on-pump group) had no 
events. Preoperative variables and operative factors with a p-value $<0.05$ in the univariate analysis were used to adjust the comparison of patency rates.

Operative factors, including the graft for target vessel anastomosis, grafting strategy, target coronary artery, revascularized myocardial territory, and sequential anastomosis, were analyzed to determine their effects on angiographic patency. All angiographic data from early, 1-year, and 5-year angiograms were integrated, and logistic regression analysis using GEE was used to consider correla- tions of multiple grafts within 1 patient. The analysis was adjusted for the time at which angiography was performed.

\section{Results}

\section{Operative data}

Off-pump CABG was performed in 75 cases (85.2\%); there were 13 conversions (14.8\%) to cardiopulmonary bypass during off-pump CABG due to hemodynamic insta-

Table 2. Operative data

\begin{tabular}{|c|c|c|c|c|}
\hline Variable & Total $(\mathrm{n}=88)$ & On-pump (n=13) & Off-pump $(n=75)$ & $\mathrm{p}$-value \\
\hline \multicolumn{5}{|l|}{ Types of surgery } \\
\hline Elective & $79(89.8)$ & $13(100.0)$ & $66(88.0)$ & 0.345 \\
\hline Urgent & $9(10.2)$ & 0 & $9(12.0)$ & 0.345 \\
\hline \multicolumn{5}{|l|}{ Intra-aortic balloon pump } \\
\hline Preoperative & $37(42.0)$ & $1(7.7)$ & $36(48.0)$ & 0.007 \\
\hline Intraoperative & $7(8.0)$ & $2(15.4)$ & $5(6.7)$ & 0.275 \\
\hline Postoperative & $1(1.1)$ & 0 & $1(1.3)$ & $>0.999$ \\
\hline Complete revascularization & $63(71.6)$ & $12(92.3)$ & $51(68.0)$ & 0.099 \\
\hline Distal anastomoses per patient & $1.9 \pm 0.8$ & $2.0 \pm 0.4$ & $1.8 \pm 0.8$ & 0.232 \\
\hline \multicolumn{5}{|l|}{ Graft usage } \\
\hline LITA & $11(7.8)$ & 0 & $11(8.9)$ & 0.270 \\
\hline RITA & $40(28.4)$ & $5(27.8)$ & $35(28.5)$ & 0.677 \\
\hline RGEA & $30(21.3)$ & 0 & $30(24.4)$ & 0.011 \\
\hline SV & $56(39.7)$ & $13(72.2)$ & $43(35.0)$ & 0.001 \\
\hline RA & $4(2.8)$ & 0 & $4(3.3)$ & 0.819 \\
\hline \multicolumn{5}{|l|}{ Grafting strategy } \\
\hline Aortocoronary & $6(4.3)$ & 0 & $6(4.9)$ & 0.596 \\
\hline Y-composite & $36(25.5)$ & $8(44.4)$ & $28(22.8)$ & 0.078 \\
\hline I-composite & $41(29.1)$ & $5(27.8)$ & $36(29.3)$ & 0.488 \\
\hline In situ & $58(41.1)$ & $5(27.8)$ & $53(43.1)$ & 0.004 \\
\hline \multicolumn{5}{|l|}{ Graft for target vessel anastomosis } \\
\hline LITA & $9(5.5)$ & 0 & $9(6.6)$ & 0.255 \\
\hline RITA & $7(4.3)$ & 0 & $7(5.1)$ & 0.377 \\
\hline RGEA & $41(25.2)$ & 0 & $41(29.9)$ & $<0.001$ \\
\hline SV & $102(62.0)$ & $26(100.0)$ & $75(54.7)$ & $<0.001$ \\
\hline RA & $5(3.1)$ & 0 & $5(3.6)$ & 0.557 \\
\hline \multicolumn{5}{|l|}{ Target native coronary artery } \\
\hline LAD & $42(25.8)$ & $2(7.7)$ & $40(29.2)$ & 0.034 \\
\hline Diagonal & $29(17.8)$ & $4(15.4)$ & $25(18.2)$ & 0.542 \\
\hline Obtuse marginal & $38(23.3)$ & $10(38.5)$ & $28(20.4)$ & 0.011 \\
\hline RCA & $2(1.2)$ & $1(3.8)$ & $1(0.7)$ & 0.231 \\
\hline Posterior descending artery & $44(27.0)$ & $6(23.1)$ & $38(27.7)$ & 0.665 \\
\hline Posterolateral branch & $8(4.9)$ & $3(11.5)$ & $5(3.6)$ & 0.089 \\
\hline \multicolumn{5}{|l|}{ Revascularized myocardial territory } \\
\hline LAD territory & $57(64.8)$ & $6(46.2)$ & $51(68.0)$ & 0.352 \\
\hline Left circumflex artery territory & $33(37.5)$ & $9(69.2)$ & $24(32.0)$ & 0.143 \\
\hline RCA territory & $51(58.0)$ & $9(69.2)$ & $42(56.0)$ & 0.841 \\
\hline
\end{tabular}

Values are presented as number (\%) or mean \pm standard deviation.

LITA, left internal thoracic artery; RITA, right internal thoracic artery; RGEA, right gastroepiploic artery; SV, saphenous vein; RA, radial artery; LAD, left anterior descending artery; RCA, right coronary artery. 
bility or poor exposure caused by severe adhesions. Twelve cases were performed in an on-pump beating fashion without aortic cross-clamping, while 1 case required aortic cross-clamping with cardioplegic arrest. Perioperative IABP support was needed in 45 patients (51.1\%). Among them, 37 patients $(42.0 \%)$ underwent IABP insertion preoperatively, 7 patients $(8.0 \%)$ received IABP support intraoperatively, and 1 patient (1.1\%) received IABP support postoperatively. Urgent surgery was performed in 9 patients (10.2\%) out of the overall cohort (Table 2).

Overall, 141 grafts were used in 88 redo CABG patients. In terms of graft usage, the saphenous vein (SV) was the most frequently used graft (63.3\%), followed by right internal thoracic artery (RITA) (46.7\%), right gastroepiploic artery (RGEA) (33.3\%), left internal thoracic artery (LITA) (11.1\%), and radial artery (RA) (5.6\%). In terms of the grafting strategy, in situ grafting was the most frequently used strategy (41.7\%), followed by I-composite grafting (29.2\%), Y-composite grafting (25.0\%), and aortocoronary grafting (4.2\%).

A total of 164 anastomoses $(1.9 \pm 0.8$ distal anastomoses

A

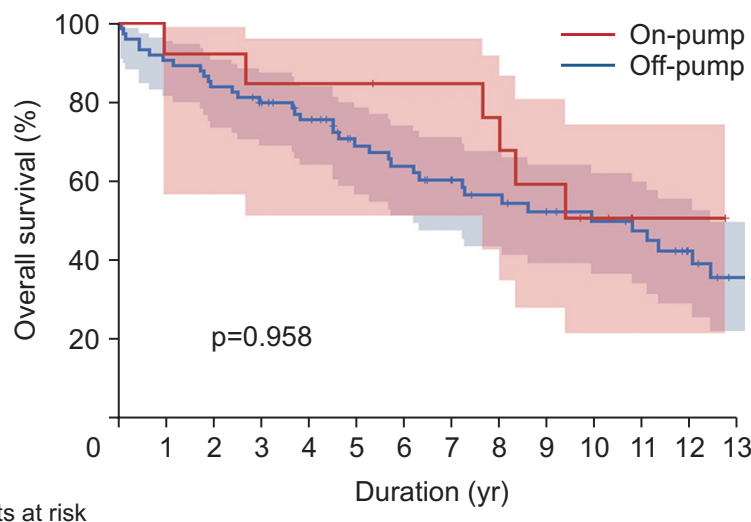

Patients at risk

$\begin{array}{lllllllllllllll}\text { On-pump } & 13 & 12 & 12 & 11 & 11 & 11 & 10 & 10 & 9 & 7 & 5 & 1 & 1 & 0\end{array}$ $\begin{array}{lllllllllllllll}\text { Off-pump } & 75 & 68 & 63 & 58 & 51 & 40 & 37 & 33 & 28 & 24 & 21 & 19 & 13 & 8\end{array}$

C

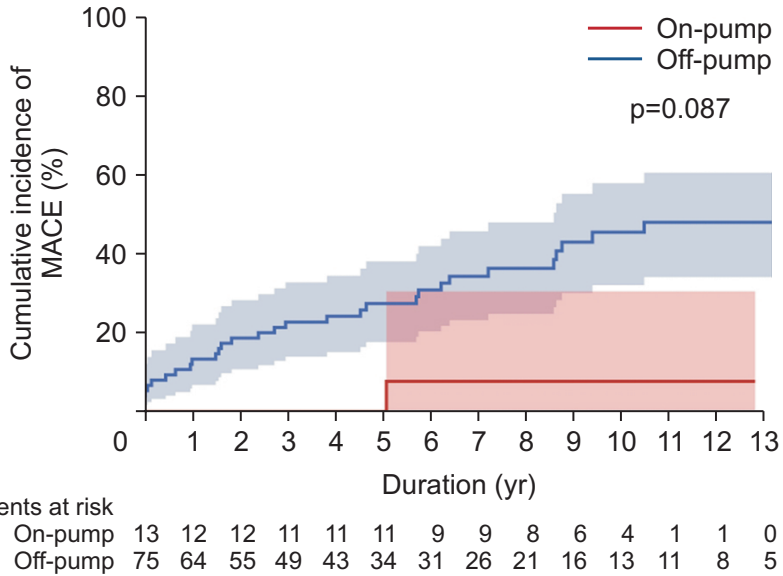

per patient) were performed in the overall study population. Anastomoses were performed with the SV (62.0\%), RGEA (25.2\%), LITA (5.5\%), RITA (4.3\%), and RA (3.1\%). The target coronary arteries were the LAD (25.8\%), diagonal branch (17.8\%), OM (23.3\%), RCA (1.2\%), PDA (27.0\%), and PLB (4.9\%). The LAD territory was revascularized in 57 patients, the LCx territory was revascularized in 33 patients, and the RCA territory was revascularized in 51 patients (Table 2).

\section{Early clinical outcomes}

There was only 1 case of operative mortality (1.1\%) among the study population; this event did not occur within 30 postoperative days. The postoperative morbidities were atrial fibrillation (30.0\%), acute kidney injury (9.1\%), reoperation for bleeding (3.4\%), stroke (3.4\%), and mediastinitis (1.1\%). The patients were extubated at 19 hours (IQR, 17-24 days) and stayed in the intensive care unit (ICU) for 24 hours (IQR, 21-45 hours). The patients were discharged on postoperative day 8 (IQR, 7-14 days). When the patients

B

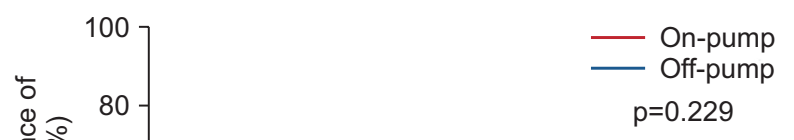

$\begin{array}{lllllllllllllll}\text { On-pump } & 13 & 12 & 12 & 11 & 11 & 11 & 10 & 10 & 9 & 7 & 5 & 1 & 1 & 0\end{array}$ $\begin{array}{lllllllllllllll}\text { Off-pump } & 75 & 68 & 63 & 58 & 51 & 40 & 37 & 33 & 28 & 24 & 21 & 19 & 13 & 8\end{array}$

Fig. 2. (A) Comparison of overall survival between the on-pump group and off-pump group in the study population. (B) Comparison of the cumulative incidence of cardiac death between the on-pump group and off-pump group. (C) Comparison of the cumulative incidence of MACE between the on-pump group and off-pump group. MACE, major adverse cardiac events. 
were classified into off-pump versus on-pump groups, there was no difference regarding mortality or morbidities between the groups. However, hospital course-related variables, including extubation time, ICU stay, and hospital stay, were longer in the on-pump group than in the offpump group, although statistical significance was only found for ICU stay (Supplementary Table 1).

\section{Mid-term clinical outcomes}

During the mid-term follow-up, all-cause mortality occurred in 45 patients (51.1\%), including 18 cardiac deaths. Overall survival, cumulative incidence of cardiac death, and cumulative incidence of MACE were $71.3 \%, 12.0 \%$, and $23.3 \%$ at 5 years after surgery, respectively. There were

Table 3. Multivariable analysis of factors associated with cardiac death

\begin{tabular}{llc}
\hline \multicolumn{1}{c}{ Variable } & $\begin{array}{c}\text { Hazard ratio }(95 \% \\
\text { confidence interval) }\end{array}$ & p-value \\
\hline Off-pump group & $6.14(0.81-786.82)$ & 0.229 \\
Age (yr) & $1.08(1.01-1.17)$ & 0.030 \\
Urgent operation & $2.06(0.62-5.62)$ & 0.208 \\
Incomplete revascularization & $2.65(0.96-7.14)$ & 0.060 \\
\hline
\end{tabular}

no significant differences in overall survival ( $\mathrm{p}=0.958)$, cumulative incidence of cardiac death $(\mathrm{p}=0.229)$, and MACE ( $\mathrm{p}=$ 0.087 ) between the on-pump and off-pump groups (Fig. 2).

Multivariable analysis revealed that age (hazard ratio [HR], 1.07; $\mathrm{p}=0.005)$ and chronic kidney disease (HR, 3.85; $\mathrm{p}=0.001)$ were risk factors for all-cause mortality, and age (HR, 1.08; $\mathrm{p}=0.030$ ) was a risk factor for cardiac death (Table 3, Supplementary Tables 2-4). No statistically significant risk factors were found for MACE (Supplementary Tables 5,6$)$.

\section{Early, 1-year, and 5-year angiographic patency}

Early, 1-year, and 5-year postoperative angiograms were obtained from $100 \%, 87.5 \%$, and $54.5 \%$ of the study patients, respectively, and demonstrated $95.7 \%, 90.1 \%$, and $92.2 \%$ overall patency rates (Table 4 ). There was no evidence that overall patency rates were different between the on-pump and off-pump groups. Aortocoronary grafting (among 4 grafting strategies), the RCA territory (among the 3 myocardial territories), and nonsequential anastomosis appeared to be associated with inferior angiographic patency, although the results failed to reach statistical significance (Table 5).

Table 4. Early, 1-year, and 5-year angiographic patency rates

\begin{tabular}{|c|c|c|c|c|}
\hline Variable & Total $(n=88)$ & On-pump $(n=13)$ & Off-pump (n=75) & $\mathrm{p}$-value \\
\hline Early & $100.0(88)$ & $100.0(13)$ & $100.0(75)$ & \\
\hline CT angiography & $1.1(1)$ & 0 & $1.3(1)$ & \\
\hline Overall patency rate & $95.7(156 / 163)$ & $100.0(26 / 26)$ & $94.9(130 / 137)$ & 0.150 \\
\hline Bilateral ITAs & $100.0(16 / 16)$ & - & $100.0(16 / 16)$ & - \\
\hline RGEA & $100.0(41 / 41)$ & - & $100.0(41 / 41)$ & - \\
\hline SV & $93.1(94 / 101)$ & $100.0(26 / 26)$ & $90.7(68 / 75)$ & 0.130 \\
\hline RA & $100.0(5 / 5)$ & - & $100.0(5 / 5)$ & - \\
\hline 1-Year & $87.5(77)$ & $92.3(12)$ & $86.7(65)$ & \\
\hline CT angiography & $32.5(25)$ & $50.0(6)$ & $29.2(19)$ & \\
\hline Overall patency rate & $90.1(128 / 142)$ & $87.5(21 / 24)$ & $90.7(107 / 118)$ & 0.630 \\
\hline Bilateral ITAs & $93.3(14 / 15)$ & - & $93.3(14 / 15)$ & - \\
\hline RGEA & $100.0(34 / 34)$ & - & $100.0(34 / 34)$ & - \\
\hline SV & $85.6(77 / 90)$ & $87.5(21 / 24)$ & $84.8(56 / 66)$ & 0.630 \\
\hline RA & $100.0(3 / 3)$ & - & $100.0(3 / 3)$ & - \\
\hline 5-Year & $54.5(48)$ & $84.6(11)$ & $49.3(37)$ & \\
\hline CT angiography & $41.7(20)$ & $63.6(7)$ & $35.1(13)$ & \\
\hline Overall patency rate & $92.2(83 / 90)$ & $86.4(19 / 22)$ & $94.1(64 / 68)$ & 0.264 \\
\hline Bilateral ITAs & $92.9(13 / 14)$ & - & $92.9(13 / 14)$ & - \\
\hline RGEA & $93.1(27 / 29)$ & - & $93.1(27 / 29)$ & - \\
\hline SV & $91.3(42 / 46)$ & $86.4(19 / 22)$ & $95.8(23 / 24)$ & 0.462 \\
\hline RA & $100.0(1 / 1)$ & - & $100.0(1 / 1)$ & - \\
\hline
\end{tabular}

Values are presented as \% (number).

CT, computed tomography; ITA, internal thoracic artery; RGEA, right gastroepiploic artery; SV, saphenous vein; RA, radial artery. 
Table 5. Relationships between operative factors and overall patency rates

\begin{tabular}{lcc}
\hline \multicolumn{1}{c}{ Variable } & Angiographic patency & p-value \\
\hline Graft for target vessel anastomosis & & - \\
Left internal thoracic artery & $100.0(29 / 29)$ & \\
Right internal thoracic artery & $87.0(20 / 23)$ & \\
Right gastroepiploic artery & $97.6(122 / 125)$ & \\
Saphenous vein & $89.0(219 / 246)$ & \\
Radial artery & $100.0(10 / 10)$ & \\
Grafting strategy & & \\
Aortocoronary & $82.4(14 / 17)$ & \\
Y-composite & $86.3(138 / 160)$ & \\
I-composite & $97.5(192 / 197)$ & \\
In situ & $94.9(56 / 59)$ & \\
Target native coronary artery & & \\
LAD & $93.3(98 / 105)$ & \\
Diagonal & $92.6(75 / 81)$ & \\
Obtuse marginal & $95.2(100 / 105)$ & \\
RCA & $100.0(5 / 5)$ & \\
Posterior descending artery & $87.5(105 / 120)$ & \\
Posterolateral branch & $100.0(17 / 17)$ & \\
Revascularized myocardial territory & & \\
LAD territory & $93.0(173 / 186)$ & \\
Left circumflex artery territory & $95.2(100 / 105)$ & \\
RCA territory & $89.4(127 / 142)$ & \\
Sequential anastomosis & & \\
No & $90.5(239 / 264)$ & \\
Yes & $95.3(161 / 169)$ & \\
\hline
\end{tabular}

Values are presented as $\%$ (number).

LAD, left anterior descending artery; RCA, right coronary artery.

\section{Discussion}

The present study demonstrated 3 main findings. First, the early and mid-term outcomes of redo CABG were excellent compared with those of other previously published studies. Second, the angiographic patency results, which were comparable with the outcomes generally observed in first-time CABG, were also acceptable. Third, early outcomes, mid-term outcomes, and angiographic patency rates were comparable between the on-pump and off-pump redo CABG groups.

In the present study, operative mortality was $1.1 \%$, whereas it was reported to be between $4 \%$ and $6 \%$ in previous studies $[6,15,16]$. In addition to the competent surgical technique and perioperative medical management, active support with IABP might have led to the excellent early outcomes observed. In this study, more than $40 \%$ of patients were supported with IABP as early as the preoperative period, which is a larger percentage than reported in other studies. Yau et al. [10] reported rates of $6.8 \%$ for 30 day mortality and $16.1 \%$ for IABP insertion in their redo
CABG population, and Maltais et al. [17] reported rates of $6 \%$ for in-hospital mortality and 20\% for IABP application. In general, patients with de novo coronary lesions or angina after CABG tend to be initially managed with medical therapy or PCI. If patients are not suitable to receive PCI due to complex lesions or if patients manifest with progressive myocardial ischemia, such as unstable vital signs, ongoing ischemia, or intractable angina, they are designated to undergo redo CABG; in such cases, medical stabilization with the aid of IABP is preferred at our institution rather than performing emergent surgery, and this strategy has been mostly successful for achieving satisfactory postoperative outcomes. Although patients who require preoperative IABP are generally in a worse condition than those who do not, IABP support provides major advantages for the operation and postoperative course. Intraoperatively, IABP enables further manipulation of the heart with acceptable hemodynamics. If hemodynamic instability occurred during the operation, we usually inserted the IABP before conversion to cardiopulmonary bypass. The frequency of intraoperative IABP therapy during redo CABG at our institution was $8.0 \%$, which was higher than the proportion of intraoperative IABP therapy during firsttime CABG (2.4\%). IABP also guarantees an uneventful postoperative course by giving the myocardium more time to recover from perioperative ischemia and reperfusion injury. Aggressive perioperative management with IABP might have been a factor that influenced our excellent early outcomes.

Previous studies reported mid-term survival at 5 years to be between $60 \%$ and $85 \%$ [15-19], and 1 study stated that the 10 -year overall survival rate was approximately $68 \%$ in redo CABG patients [20]. The present study showed a $71.3 \% 5$-year overall survival rate, which is consistent with previous studies.

Most previous studies did not investigate the culprits for reoperation; our study showed that stenosis or occlusion of a previous graft alone accounted for approximately twothirds of the reoperations, while progression of native vessel lesions alone and mixed (graft and native vessel) lesions each accounted for approximately one-sixth of the reoperations. We could infer that advances in graft selection, surgical techniques, and medical management will reduce the need for redo CABG by improving graft patency and preventing the occurrence of culprit lesions.

Off-pump redo CABG has been widely regarded to be comparable or better than on-pump redo CABG in terms of early outcomes. If off-pump CABG is technically feasible, it has many strengths compared with on-pump CABG. 
In patients with marginal cardiac function, off-pump CABG, which needs neither aortic cross-clamping nor cardioplegic arrest, would be beneficial in terms of myocardial protection. A lower dose of heparin during off-pump CABG than on-pump CABG would lead to fewer postoperative bleeding complications. Indeed, previous studies showed a trend toward a decrease in mortality and morbidity in the off-pump redo CABG groups. Dohi et al. [21] reported a $13.6 \%$ p reduction $(22.9 \%$ versus $9.3 \%)$ in the composite 30 -day mortality or major morbidity rate in the unadjusted study population and a $10.5 \%$ p reduction $(21.5 \%$ versus $11 \%$ ) in the adjusted population after propensity matching; both of these results favor off-pump redo CABG. Bruno et al. [20] showed a reduction in the composite of in-hospital mortality, acute kidney injury, stroke, and severe low cardiac output requiring IABP in the off-pump group.

In this study, mid-term outcomes, including overall survival, cardiac death, and MACE, showed no significant difference between the off-pump and on-pump redo CABG groups. These findings are consistent with a previous study that showed that the mid-term survival of off-pump redo CABG was comparable to that of on-pump redo CABG [20]. However, we found a tendency toward a higher incidence of MACE in the off-pump group in our study, and we speculate that this finding might originate from the achievement of complete revascularization.

Completeness of coronary revascularization has been widely known to be associated with long-term outcomes $[16,22,23]$; thus, in general, complete revascularization is the goal of CABG. In most studies, the rate of incomplete revascularization has been reported to be higher in the offpump redo $C A B G$ group than in the on-pump redo $C A B G$ group [20,22-24]. Similarly, in the operative data of the present study, complete revascularization and LCx territory revascularization were achieved more frequently in the on-pump group. In our patients, if lateral wall revascularization was indispensable, on-pump conversion was chosen when it was difficult to achieve adhesiolysis, exposure, and anastomosis without cardiopulmonary bypass support. However, if a limited approach without cardiopulmonary bypass, such as the transabdominal approach for RGEA to PDA, was planned, only the culprit lesion was revascularized, and intermediate lesions were left without intervention, resulting in incomplete revascularization. In terms of complete revascularization, these findings might have reflected the tendency toward better long-term outcomes in the on-pump group.

In the multivariable analysis, age and chronic kidney disease were identified as risk factors for all-cause mortality, but the clinical significance of this finding is limited because these are universal risk factors for mortality after any type of cardiac surgery.

There are several limitations to this study. First, this was a retrospective observational study with a small sample size at a single center; hence, statistically significant differences were difficult to derive because of the small study population. Second, the patient cohort was formed over a long time period; thus, many confounding factors, such as changes in clinical practice over time, might have influenced the study results. Third, an assessment of the severity of coronary disease was not performed. Fourth, angiographic patency rates were most likely underestimated considering the number of patients who did not undergo follow-up angiography. Fifth, graft usage was not included in the risk factor analysis for mid-term clinical outcomes. Sixth, operative techniques according to the kind of inflow graft were not considered for the analysis of clinical outcomes and angiographic patency. Finally, the comparison between the off-pump and on-pump groups may have been affected by bias because the conversion from off-pump to on-pump took place during the procedure freeing the heart from adhesions, and these selective patients were included in the on-pump group for the final analysis.

In conclusion, despite surgical complexity and underlying comorbidities, redo CABG demonstrated low operative mortality and morbidity, with excellent mid-term outcomes and angiographic patency. The comparison between on-pump and off-pump redo CABG showed no significant difference in early and mid-term outcomes. A surgeon does not need to hesitate to perform cardiopulmonary bypass when the technical complexity occurs during redo $\mathrm{CABG}$ procedures.

\section{Conflict of interest}

No potential conflict of interest relevant to this article was reported.

\section{Acknowledgments}

The authors thank the Medical Research Collaborating Center, Seoul National University Hospital for their statistical support.

\section{ORCID}

Suk Ho Sohn: https://orcid.org/0000-0001-7391-3415 
Seung Hyun Kim: https://orcid.org/0000-0003-3999-8229

Ho Young Hwang: https://orcid.org/0000-0002-8935-8118

Ki-Bong Kim: https://orcid.org/0000-0002-4918-7262

\section{Supplementary materials}

Supplementary materials can be found via https://doi. org/10.5090/jcs.20.122. Supplementary Table 1. Early clinical outcomes. Supplementary Table 2. Univariate analysis of factors associated with all-cause mortality. Supplementary Table 3. Multivariable analysis of factors associated with all-cause mortality. Supplementary Table 4. Univariate analysis of factors associated with cardiac death. Supplementary Table 5. Univariate analysis of factors associated with major adverse cardiac events. Supplementary Table 6. Multivariable analysis of factors associated with major adverse cardiac events.

\section{References}

1. Carey JS, Cukingnan RA, Singer LK. Quality of life after myocardial revascularization: effect of increasing age. J Thorac Cardiovasc Surg 1992;103:108-15.

2. Cosgrove DM, Loop FD, Lytle BW, et al. Predictors of reoperation after myocardial revascularization. J Thorac Cardiovasc Surg 1986; 92:811-21

3. Hillis LD, Smith PK, Anderson JL, et al. 2011 ACCF/AHA guideline for coronary artery bypass graft surgery: executive summary: a report of the American College of Cardiology Foundation/American Heart Association Task Force on Practice Guidelines. Circulation 2011; 124:2610-42.

4. Authors/Task Force members, Windecker S, Kolh P, et al. 2014 ESC/ EACTS guidelines on myocardial revascularization: the Task Force on Myocardial Revascularization of the European Society of Cardiology (ESC) and the European Association for Cardio-Thoracic Surgery (EACTS) developed with the special contribution of the European Association of Percutaneous Cardiovascular Interventions (EAPCI). Eur Heart J 2014;35:2541-619.

5. Sousa-Uva M, Neumann FJ, Ahlsson A, et al. 2018 ESC/EACTS guidelines on myocardial revascularization. Eur J Cardiothorac Surg 2019;55:4-90.

6. Ghanta RK, Kaneko T, Gammie JS, Sheng S, Aranki SF. Evolving trends of reoperative coronary artery bypass grafting: an analysis of the Society of Thoracic Surgeons Adult Cardiac Surgery Database. J Thorac Cardiovasc Surg 2013;145:364-72.

7. Sabik JF 3rd, Blackstone EH, Houghtaling PL, Walts PA, Lytle BW. Is reoperation still a risk factor in coronary artery bypass surgery? Ann Thorac Surg 2005;80:1719-27.

8. Rufa MI, Ursulescu A, Nagib R, et al. Off-pump versus on-pump redo coronary artery bypass grafting: a propensity score analysis of long-term follow-up. J Thorac Cardiovasc Surg 2020;159:447-56.

9. Brilakis ES, Held C, Meier B, et al. Effect of ticagrelor on the outcomes of patients with prior coronary artery bypass graft surgery: insights from the PLATelet inhibition and patient outcomes (PLATO) trial. Am Heart J 2013;166:474-80.

10. Yau TM, Borger MA, Weisel RD, Ivanov J. The changing pattern of reoperative coronary surgery: trends in 1230 consecutive reoperations. J Thorac Cardiovasc Surg 2000;120:156-63.

11. Spiliotopoulos K, Maganti M, Brister S, Rao V. Changing pattern of reoperative coronary artery bypass grafting: a 20-year study. Ann Thorac Surg 2011;92:40-7.

12. Kim KB, Choi JW, Oh SJ, et al. Twenty-year experience with offpump coronary artery bypass grafting and early postoperative angiography. Ann Thorac Surg 2020;109:1112-9.

13. FitzGibbon GM, Burton JR, Leach AJ. Coronary bypass graft fate: angiographic grading of 1400 consecutive grafts early after operation and of 1132 after one year. Circulation 1978;57:1070-74.

14. Austin PC, Fine JP. Practical recommendations for reporting FineGray model analyses for competing risk data. Stat Med 2017;36: 4391-400.

15. Yap CH, Sposato L, Akowuah E, et al. Contemporary results show repeat coronary artery bypass grafting remains a risk factor for operative mortality. Ann Thorac Surg 2009;87:1386-91.

16. Di Mauro M, Iaco AL, Contini M, et al. Reoperative coronary artery bypass grafting: analysis of early and late outcomes. Ann Thorac Surg 2005;79:81-7.

17. Maltais S, Widmer RJ, Bell MR, et al. Reoperation for coronary artery bypass grafting surgery: outcomes and considerations for expanding interventional procedures. Ann Thorac Surg 2017;103:188692.

18. Cole JH, Jones EL, Craver JM, et al. Outcomes of repeat revascularization in diabetic patients with prior coronary surgery. J Am Coll Cardiol 2002;40:1968-75.

19. Varghese I, Samuel J, Banerjee S, Brilakis ES. Comparison of percutaneous coronary intervention in native coronary arteries vs. bypass grafts in patients with prior coronary artery bypass graft surgery. Cardiovasc Revasc Med 2009;10:103-9.

20. Bruno VD, Zakkar M, Rapetto F, et al. Early health outcome and 10year survival in patients undergoing redo coronary surgery with or without cardiopulmonary bypass: a propensity score-matched analysis. Eur J Cardiothorac Surg 2017;52:945-51.

21. Dohi M, Miyata H, Doi K, et al. The off-pump technique in redo coronary artery bypass grafting reduces mortality and major morbidities: propensity score analysis of data from the Japan Cardiovascular Surgery Database. Eur J Cardiothorac Surg 2015;47:299-308.

22. Tugtekin SM, Alexiou K, Kappert U, et al. Coronary reoperation with and without cardiopulmonary bypass. Clin Res Cardiol 2006; 95:93-8. 
23. Czerny M, Zimpfer D, Kilo J, et al. Coronary reoperations: recurrence of angina and clinical outcome with and without cardiopulmonary bypass. Ann Thorac Surg 2003;75:847-52.

24. Kara I, Cakalagaoglu C, Ay Y, et al. Reoperative coronary artery by- pass surgery: the role of on-pump and off-pump techniques on factors affecting hospital mortality and morbidity. Ann Thorac Cardiovasc Surg 2013;19:435-40. 